\title{
Expression of Matrix Metalloproteinase-7 is an Unfavorable Prognostic Factor in Intrahepatic Cholangiocarcinoma
}

\author{
Teijiro Hirashita • Yukio Iwashita • Masayuki Ohta • \\ Yoko Komori • Hidetoshi Eguchi • Kazuhiro Yada • \\ Seigo Kitano
}

Received: 30 September 2011 / Accepted: 28 December 2011 /Published online: 13 January 2012

(C) The Author(s) 2012. This article is published with open access at Springerlink.com

\begin{abstract}
Background Intrahepatic cholangiocarcinoma (IHCC) is a highly malignant neoplasm, but the prognostic factors of IHCC are not yet fully understood. The matrix metalloproteinases (MMPs) are known to be related to tumor viability. The aim of this study was to evaluate the prognostic significance of clinicopathological and immunohistochemical characteristics of resected IHCC.

Patients and Methods From 1996 to 2006, we surgically treated 35 patients with IHCC. Clinicopathological and immunohistochemical characteristics, including expression of MMPs, vascular endothelial growth factor, and epidermal growth factor receptor in the resected specimens, were investigated, and overall survival rates were evaluated with regard to the characteristics using univariate and multivariate analyses.

Results Univariate analysis revealed the significant prognostic factors to be preoperative serum CEA and CA19-9, intraoperative transfusion, tumor size, surgical margin, lymph node metastasis, invasion of portal and hepatic vein, intrahepatic metastasis, UICC stage, and expression of MMP-7. Subsequent multivariate analysis indicated that MMP-7 was an independent prognostic factor (hazard ratio (HR), 4.698; 95\% confidence interval (CI), 0.057-0.866; $P=0.03$ ) along with intrahepatic metastasis (HR, 5.694; 95\% CI, 0.029-0.706; $P=0.017$ ).

Conclusion MMP-7 expression is associated with a poor prognosis in patients with resected IHCC.
\end{abstract}

Keywords Cholangiocarcinoma $\cdot$ Matrix metalloprotease · Matrix metalloprotease- $7 \cdot$ Prognostic factor

\section{Introduction}

Intrahepatic cholangiocarcinoma (IHCC) is a highly malignant neoplasm originating from the bile duct system with markers of cholangiocyte differentiation. ${ }^{1,2}$ The incidence of IHCC is increasing worldwide, and it is the second most common form of primary liver cancer next to that of hepatocellular carcinoma. Despite advances in diagnosis and treatment, most patients present with advanced metastatic

T. Hirashita $(\bowtie) \cdot$ Y. Iwashita $\cdot$ M. Ohta $\cdot$ Y. Komori $\cdot$ H. Eguchi $\cdot$ K. Yada $\cdot$ S. Kitano

Department of Surgery I, Oita University Faculty of Medicine,

1-1 Idaigaoka, Hasama-machi,

Yufu, Oita 879-5593, Japan

e-mail: teij03@oita-u.ac.jp lesions and are not amenable to surgical extirpation or liver transplantation. $^{3-7}$ Furthermore, the current chemotherapy regimen that is used to treat IHCC offers very limited benefit in terms of patient survival. When compared with other malignancies, IHCC is generally characterized by strong proliferation, invasion, and early metastasis.

Matrix metalloproteinases (MMPs) are zinc-dependent neutral endopeptidases, which participate in degradation of extracellular matrix proteins. ${ }^{8,9}$ They play roles in many physiological processes, such as bone remodeling and organogenesis, and have additional roles in the reorganization of tissues during pathological conditions such as inflammation, invasion, and metastasis of cancer cells. ${ }^{8,10}$ Many recent studies have provided evidence that the biological activities of various cell surface molecules are proteolytically modulated by several MMPs, including MMP-2, -9, and $-7 .{ }^{11-13}$ These MMPs are likely to regulate cellular functions by activating, inactivating, or releasing membrane proteins. Such regulation of cell surface proteins, as well as MMP-catalyzed degradation 
of the extracellular matrix (ECM), a natural barrier against tumor invasion, is important to tumor invasion and metastasis.

MMP-7, the smallest of the MMPs, has broad substrate specificity and has been demonstrated to degrade or process a variety of matrix and non-matrix molecules. ${ }^{14}$ Unlike most MMPs, which are expressed by stromal cells, MMP-7 is principally expressed by epithelial cells. ${ }^{15}$ In human cholangiocarcinomas, this enzyme appears to be one of the most important MMPs because the expression of MMP-7 correlates highly with the malignant and metastatic potentials of the cancers. ${ }^{16}$

In this study, we investigated the association of clinicopathological characteristics of resected IHCCs with prognosis. To improve the prognosis in IHCC, we believe that a fuller, more detailed understanding of the prognostic factors of IHCC is required.

\section{Patients and Methods}

\section{Patients}

From 1996 to 2006, a total of 35 Japanese patients with IHCC underwent hepatectomies at the Department of Surgery I, Oita University Faculty of Medicine, Oita, Japan (Table 1). IHCC was defined as a malignancy arising from the intrahepatic bile duct; perihilar tumors were excluded. Patient age was $65 \pm 10$ years $($ mean \pm SD), and the patients included 9 women and 26 men with a female-to-male ratio of 1:2.9. All patients underwent surgery in our institution. The surgical methods used were right trisectionectomy ( $n=$ $1)$, right hepatectomy including extended right hepatectomy $(n=12)$, left hepatectomy including extended left hepatectomy $(n=18)$, and others $(n=4)$. Clinical characteristics and histopathological and immunohistochemical findings of the IHCC patients were examined retrospectively. We followed

Table 1 Patient characteristics

\begin{tabular}{ll}
\hline Number of patients & 35 \\
Age (years) & $64.9 \pm 10.3$ \\
Sex (male/female) & $26 / 9$ \\
Operative procedure & \\
Right trisectionectomy & 1 \\
Right hepatectomy & 12 \\
Left hepatectomy & 18 \\
Others & 4 \\
Operation time (min) & $463 \pm 170$ \\
Blood loss (g) & $1,190 \pm 750$ \\
Transfusion & $17(48.6 \%)$ \\
Tumor size (mm) & $58.9 \pm 31.2$ \\
Surgical margin (mm) & $5.6 \pm 7.1$ \\
Complications & $16(45.7 \%)$ \\
Postoperative hospital stay (days) & $40.2 \pm 21.6$ \\
\hline
\end{tabular}

the guidelines of the ethical principles of the World Medical Association's Declaration of Helsinki in this study.

Histopathological Assessment

Tumor staging and histological classification were assessed according to the TNM Classification of Malignant Tumours defined by the International Union Against cancer. According to the General Rules for the Clinical and Pathological Study of Primary Liver Cancer, ${ }^{17}$ macroscopic types of IHCC were classified into two groups: the mass-forming type and other types, which include the periductal and the intraductal growth types. Data for other clinicopathological factors including age, sex, preoperative serum levels of carcinoembryonic antigen (CEA) and CA19-9, operation time, blood loss, transfusion, tumor size, differentiation, lymph node metastasis, invasion of portal and hepatic vein, bile duct invasion, surgical margin, intrahepatic metastasis, and UICC stage were categorized as shown in Table 2.

\section{Immunohistochemistry}

Immunohistochemistry for MMP-2, -7, -9, vascular endothelial growth factor (VEGF), and epidermal growth factor receptor (EGFR) was performed using an Envision+system (Dako, Cytomation, Glostrup, Denmark). Sources and dilutions of primary antibodies were as follows: anti MMP-2, -7, -9 (mouse monoclonal; diluted 1:100; Daiichi Fine Chemical Co., Ltd., Toyama, Japan), anti-VEGF (mouse monoclonal; diluted 1:100; Santa Cruz Biotechnology, Inc., Santa Cruz, CA), and anti-EGFR (mouse monoclonal, diluted 1:500; Santa Cruz Biotechnology, Inc.).

Formalin-fixed, paraffin-embedded serial tissue sections (4 $\mu \mathrm{m})$ were deparaffinized and rehydrated in xylene and grade-diluted ethanol (50-100\%) and submerged for $20 \mathrm{~min}$ in $0.3 \% \mathrm{H}_{2} \mathrm{O}_{2}$ with absolute methanol to block endogenous peroxidase activity. After protein blocking, the sections were incubated with each primary antibody at room temperature for $2 \mathrm{~h}$. The sections were then treated for $20 \mathrm{~min}$ with biotinylated anti-mouse IgG (Nichirei Biosciences Inc., Tokyo, Japan), followed by treatment for $30 \mathrm{~min}$ with streptavidin-biotin-peroxidase complex (Nichirei Biosciences Inc.), at room temperature with a PBS wash between each step. The slides were developed with diaminobenzidine$\mathrm{H}_{2} \mathrm{O}_{2}$ (DAB+system, Dako Japan Inc., Tokyo, Japan). The nuclei were also lightly stained with hematoxylin.

Evaluation of Immunohistochemistry

The slides were reviewed by two independent observers who had no knowledge of patient outcomes. The expression of MMPs was evaluated semiquantitatively according to the percentage of positively stained cells: negative (positive 
Table 2 Results of univariate analysis

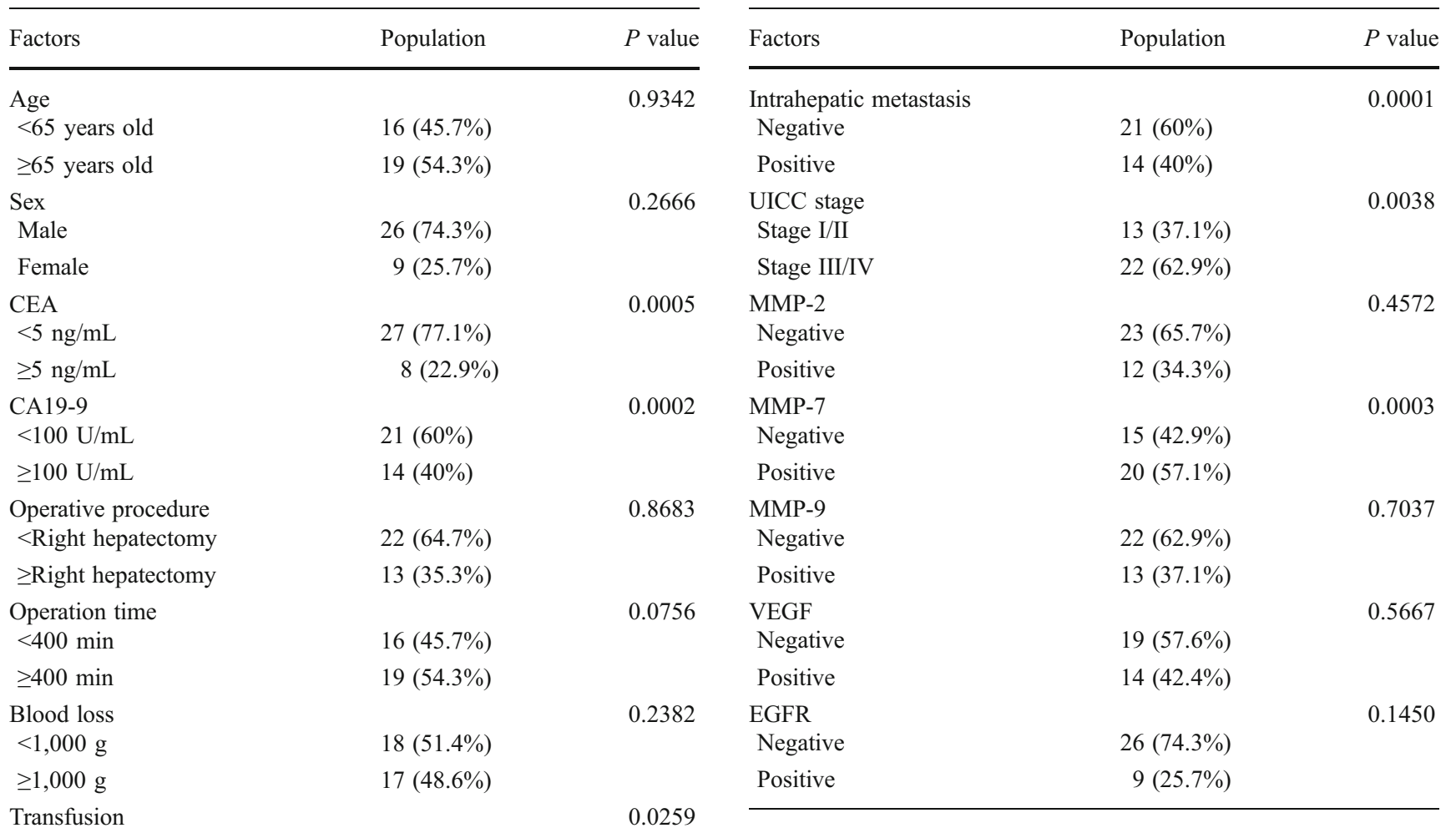

Yes

No

Tumor size (mm)

$<50 \mathrm{~mm}$

$\geq 50 \mathrm{~mm}$

Surgical margin

Negative

Positive

Complications

Yes

No

Macroscopic type

Mass forming

Non-mass forming

Differentiation

Well to moderate

Poor

Lymph node metastasis

Negative

Positive

Invasion of portal vein

Negative

Positive

Invasion of hepatic vein

Negative

Positive

Bile duct invasion

Negative

Positive
$17(48.6 \%)$

$18(51.4 \%)$

$17(48.6 \%)$

$18(51.4 \%)$

$31(88.6 \%)$

$4(11.4 \%)$

$16(45.7 \%)$

$19(54.3 \%)$

$28(80 \%)$

$7(20 \%)$

$22(62.9 \%)$

$13(37.1 \%)$

$25(71.4 \%)$

$10(28.6 \%)$

$20(57.1 \%)$

$15(42.9 \%)$

$25(71.4 \%)$

$10(28.6 \%)$

$15(45.5 \%)$

$18(54.6 \%)$ tumor cells/total tumor cells $\leq 10 \%$ ) and positive (positive tumor cells/total tumor cells $>10 \%$ ). The expression of MMPs in the stromal and inflammatory cells was not evaluated in this study. The expression of VEGF was defined as follows: negative (positive tumor cells/total tumor cells $\leq 30 \%$ ) and positive (positive tumor cells/total tumor cells $>30 \%$ ). The expression of EGFR was defined as follows: 0.7916 negative (positive tumor cells/total tumor cells $\leq 10 \%$ ) and positive (positive tumor cells/total tumor cells $>10 \%$ ).
0.6720

0.3813

0.0001

0.0072

0.0067

0.7648

\section{Statistical Analysis}

Results are expressed as means \pm SD. Postoperative survival probabilities were calculated by the Kaplan-Meier method and analyzed by the log-rank test. Difference was regarded as significant when the $P$ value was less than 0.05 . Multivariate analyses were performed using the factors identified as significant by univariate analyses. All statistical analyses were performed using Dr SPSS II software (version $11.01 \mathrm{~J}$; SPSS Japan Inc., Tokyo, Japan).

\section{Results}

Overall Survival and Results of Univariate Analysis

Overall 2- and 5-year survival rates were $50.1 \%$ and $43.3 \%$, respectively (Fig. 1). Results of the univariate analysis 
$(\%)$

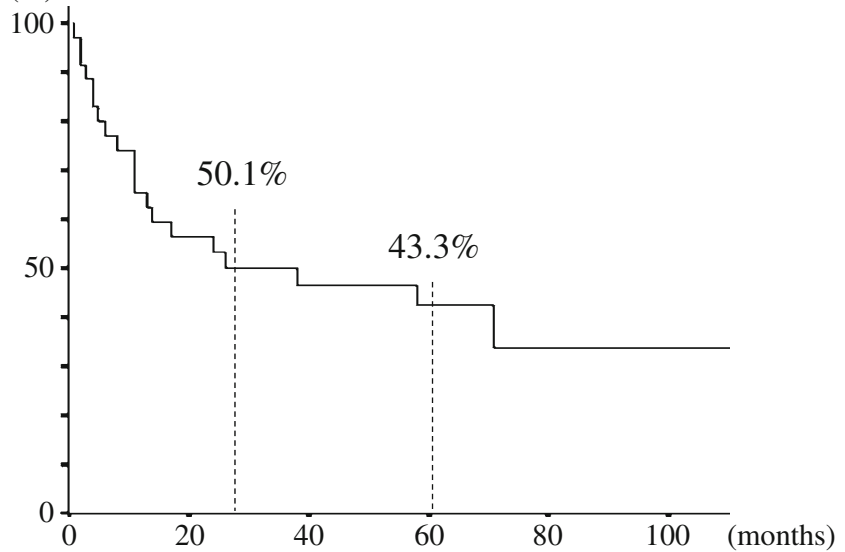

Fig. 1 Cumulative survival curves in 35 patients with resected IHCC. Survival rates at 2 and 5 years after operation were $50.1 \%$ and $43.3 \%$, respectively

of prognostic factors for overall survival are listed in Table 2. The univariate analysis revealed the statistically significant prognostic factors among the clinicopathological characteristics to be preoperative serum levels of CEA and CA19-9, intraoperative transfusion, tumor size, surgical margin, lymph node metastasis, invasion of portal and hepatic veins, intrahepatic metastasis, and UICC stage.

Immunohistochemical results of expression rates of MMP-2, -7, -9; VEGF; and EGFR and the univariate analysis of prognostic factors for overall survival are also listed in Table 2. MMP-2, -7, -9, VEGF, and EGFR were expressed in $23(65.7 \%), 15$ (42.9\%), $22(62.9 \%), 19$ $(57.6 \%)$, and $26(74.3 \%)$ of the 35 IHCC patients, respectively. Univariate analysis revealed the statistically significant prognostic factor among immunohistochemical findings to be MMP-7. The 5-year survival rates of MMP$7(+)$ and MMP-7(-) patients were $72.7 \%$ and $18.3 \%$, respectively (Fig. 2a). Positively stained cancer cells were distributed heterogeneously in the tumor nests. Carcinoma cell cytoplasm was stained brown for MMP-7, but stromal cells (other than some monocytes or surrounding normal mucosa) were not stained (Fig. 2b, c)

\section{Results of Multivariate Analysis}

In this study, 12 factors including MMP-7 expression were identified as significant prognostic factors by univariate analysis. Multivariate analysis, using these 12 factors, revealed that MMP-7 expression was an independent prognostic factor (hazard ratio [HR], 4.698; 95\% confidence interval [CI], 0.057-0.866; $P=0.03$ ) along with intrahepatic metastasis (HR, 5.694; 95\% CI, 0.029-0.706; $P=0.017$; Table 3). Lymph node metastasis showed a tendency to indicate poor prognosis; however, it was not a statistically significant indicator (HR, 3.426; 95\% CI, 0.086-1.073; $P=0.064)$.

\section{Discussion}

Many clinicopathological factors, such as lymph node metastasis, UICC stage, surgical margin, R0 resection, cirrhosis, use of postoperative adjuvant chemotherapy, and other factors, are potential prognostic factors after resection of IHCC. ${ }^{18-21}$ However, there has been no definitive way to predict prognosis of IHCC using molecules. This study was performed to determine whether expression of MMP-2, -7, -9, VEGF, and EGFR in resected specimens of IHCC can predict disease outcome. As a result, MMP-7 expression in the tumor cells was found to be a prognostic factor, in addition to that of intrahepatic metastasis.

Recently, many targeted therapies against EGFR, VEGF, and human EGFR type 2 (HER2) such as cetuximab, lapatinib, erlotinib, and bevacizumab have been used for treatment of gastrointestinal cancers. Development of targeted agents in biliary tract cancer (BTC) including IHCC has lagged behind other types of tumors, and there are a few phase II studies examining early experience of efficacy and safety of targeted therapies for BTC patients. ${ }^{22-26}$ Chemotherapy has been the main therapeutic modality in locally advanced or metastatic BTC, and a randomized, controlled, phase III trial of 410 patients with BTCs has established the combination of gemcitabine and cisplatin as a new global standard for the treatment of locally advanced or metastatic BTC. ${ }^{27}$ Although the results are encouraging, the study strongly suggests that BTC remains very difficult to treat with current methods, and molecular targeted therapy is urgently needed for this deadly disease. According to phase II studies of the targeted therapies, cetuximab may be useful for BTC when combined with gemcitabine and oxaliplatin. Gruenberger et al. ${ }^{22}$ reported that cetuximab plus gemcitabine/oxaliplatin (GEMOX) was well tolerated and had encouraging antitumor activity, leading to resection in one third of patients. These findings warrant further study of cetuximab plus GEMOX in a large randomized trial. In the present study, the rate of positivity for EGFR was $74.3 \%$, and the 5-year survival rates of $\operatorname{EGFR}(+)$ and $\operatorname{EGFR}(-)$ patients were $45.9 \%$ and $33.3 \%$, respectively. Univariate analysis revealed that $\operatorname{EGFR}(+)$ patients had a tendency for poor prognosis; however, the difference between $\operatorname{EGFR}(+)$ and EGFR $(-)$ patients was not statistically significant $(P=0.145)$.

MMPs can collectively degrade all components of the ECM and play important roles in embryo development, morphogenesis, tissue remodeling, as well as tumor invasion and metastasis. ${ }^{28,29}$ With regard to tumor invasion, the extracellular proteases, especially MMPs, appear to be 
Fig. 2 a Cumulative survival curves in IHCC patients with or without expression of matrix metalloproteinase-7 (MMP-7). The 5-year survival rates of the patients with and without the expression of MMP-7 were $72.7 \%$ and $18.3 \%$, respectively. The log-rank test revealed statistically significant differences between the two groups ( $P=0.0003)$. Immunostaining for MMP-7 in tumor tissues: $\mathbf{b}$ positive and $\mathbf{c}$ negative staining in cancer cells. In the positive staining, cytoplasm of the cancer cells was stained brown for MMP-7, but stromal cells were not stained a

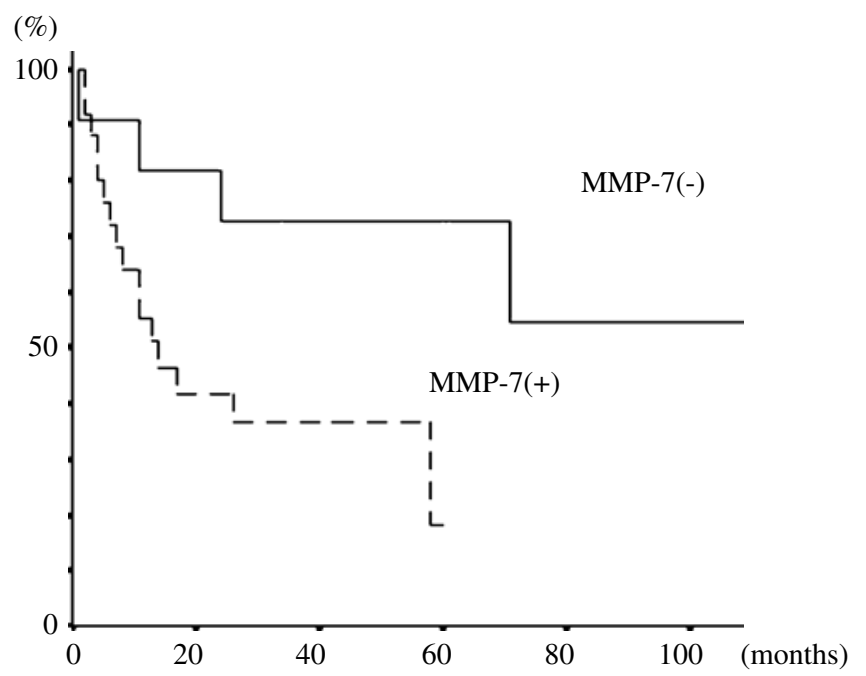

b

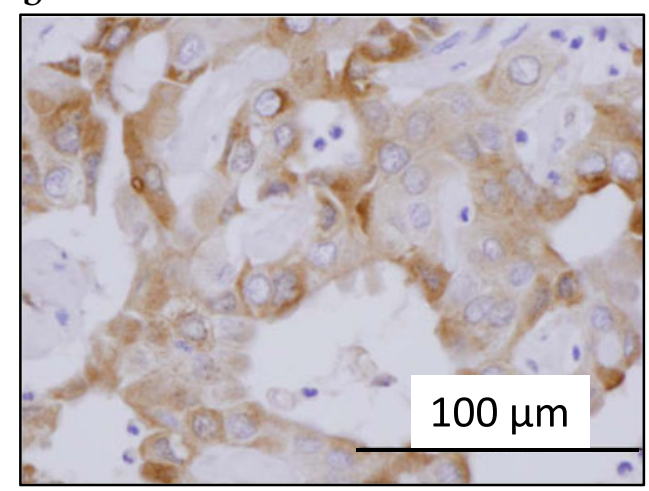

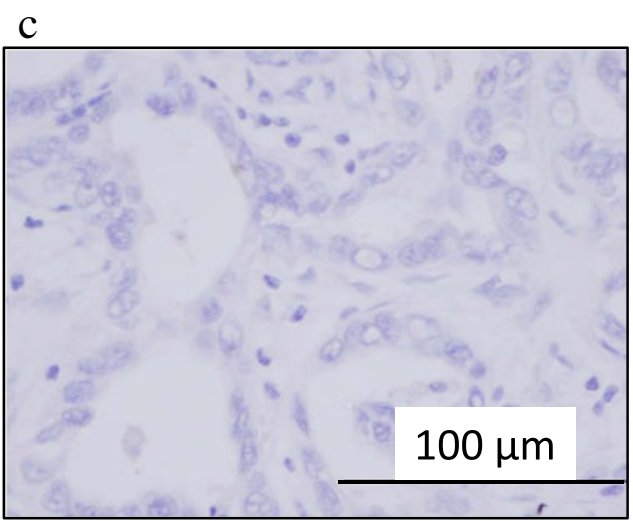

crucial for this process. In fact, the invasion of cancer cells into nearby stroma, across blood vessel walls, and through normal epithelial cell layers has been shown to be promoted by various MMPs. ${ }^{28-30}$ Among the MMPs, MMP-7 is wellknown for its involvement with many malignant tumors. MMP-7 is associated with malignancies in the colon, pancreas, and biliary tract. ${ }^{31,32}$ By degrading ECM components

Table 3 Results of multivariate analyses regarding overall survival

\begin{tabular}{lccc}
\hline & HR & $95 \%$ CI & $P$ value \\
\hline CEA & 0.001 & $0.242-4.299$ & 0.978 \\
CA19-9 & 1.270 & $0.182-1.583$ & 0.260 \\
Transfusion & 1.184 & $0.211-1.523$ & 0.271 \\
Tumor size $(<50, \geq 50 \mathrm{~mm})$ & 0.364 & $0.57-1.866$ & 0.546 \\
Surgical margin & 1.116 & $0.45-14.384$ & 0.291 \\
Lymph node metastasis & 3.426 & $0.086-1.073$ & 0.064 \\
Invasion of portal vein & 0.422 & $0.12-2.9$ & 0.516 \\
Invasion of hepatic vein & 0.018 & $0.292-4.103$ & 0.894 \\
Intrahepatic metastasis & 5.694 & $0.029-0.706$ & 0.017 \\
UICC stage & 0.006 & $0.175-6.594$ & 0.938 \\
MMP-7 & 4.698 & $0.057-0.866$ & 0.030 \\
\hline
\end{tabular}

such as type-IV collagen, fibronectin, and laminin, MMP-7 plays important roles in invasion and metastasis of carcinoma cells. ${ }^{33}$ Interestingly, cleavage of cell surface proteins other than ECM components by this MMP may also contribute to tumorigenic effects. For example, MMP-7 activates EGFR by releasing a ligand of epidermal growth factor and tumor growth factor- $\alpha .{ }^{34}$ Moreover, by processing E-cadherin and thereby inducing loose and then tight aggregation of tumor cells, this proteinase induces homotypic adhesion of human colorectal cancer cells and enhances the metastatic potential in vivo. ${ }^{35}$ By disrupting tight junction structure and a consequent induction of cell dissociation, MMP-7 might enhance metastasis as well as invasion of IHCC cells. ${ }^{36}$

Some in vitro and in vivo studies have been performed to determine how MMPs contribute to progression of BTC including IHCC. It has been reported that the serum level of MMP-7 is elevated in BTC patients, and this appears to have a potential to differentiate BTC from benign biliary tract diseases. ${ }^{37,38}$ Moreover, histological expression of MMP-7 has been reported to be an unfavorable postoperative prognostic factor of BTC. ${ }^{39,40}$ Also, higher expression of MMP-9 is suggested to be associated with the potential of 
invasion $^{41}$; however, MMP-9 has not been reported to be a prognostic factor. With regard to MMP-2, it is controversial whether this MMP is associated with poor prognosis. ${ }^{41,42}$ The multivariate analysis in the present study showed that immunohistochemical expression of MMP-7 in IHCC tissues was associated with poor prognosis, and expression of MMP-2 and -9 was not associated with it. This is the first report, to our knowledge, in which multivariate analysis shows the clinical significance of MMP-7 expression in patients with IHCC. In this study, the Kaplan-Meier life table indicated that 5 -year survival was 4 times greater in patients without MMP-7-expressing tumors compared with that in patients with MMP-7-expressing tumors $(72.7 \%$ vs. $18.3 \%$ ). Therefore, we believe that patients with MMP-7 expressing tumors of IHCC will have poorer prognoses and should be followed up more closely after the operation. In addition, MMP-7 could be a potential therapeutic target. If specific inhibitor of MMP-7 expression by small RNA and so on is developed in the future, ${ }^{43}$ it may be useful to improve the prognosis in combination with standard chemotherapy.

In conclusion, the present study suggested that MMP-7 is associated with a poor prognosis in IHCC patients. However, this study included a small number of patients, and further studies enrolling a larger number of patients will be required to confirm the results of this study.

Conflict of interest All authors declare no conflicts of interest.

Financial support No financial support was received for this study.

Open Access This article is distributed under the terms of the Creative Commons Attribution Noncommercial License which permits any noncommercial use, distribution, and reproduction in any medium, provided the original author(s) and source are credited.

\section{References}

1. de Groen PC, Gores GJ, LaRusso NF, Gunderson LL, Nagorney DM. Biliary tract cancers. N Engl J Med 1999;341:1368-1378.

2. Roberts SK, Ludwig J, Larusso NF. The pathobiology of biliary epithelia. Gastroenterology 1997;112:269-279.

3. Blechacz B, Gores GJ. Cholangiocarcinoma: advances in pathogenesis, diagnosis, and treatment. Hepatology 2008;48:308-321.

4. Blechacz BR, Gores GJ. Cholangiocarcinoma. Clin Liver Dis 2008;12:131-150.

5. Nathan H, Pawlik TM, Wolfgang CL, Choti MA, Cameron JL, Schulick RD. Trends in survival after surgery for cholangiocarcinoma: a 30-year population-based SEER database analysis. J Gastrointest Surg 2007;11:1488-1496.

6. Patel T. Increasing incidence and mortality of primary intrahepatic cholangiocarcinoma in the United States. Hepatology 2001;33:13531357.
7. Olnes MJ, Erlich R. A review and update on cholangiocarcinoma. Oncology 2004;66:167-179

8. Egeblad M, Werb Z. New functions for the matrix metalloproteinases in cancer progression. Nat Rev Cancer 2002;2:161-174.

9. Garg P, Sarma D, Jeppsson S, Patel NR, Gewirtz AT, Merlin D, Sitaraman SV. Matrix metalloproteinase-9 functions as a tumor suppressor in colitis-associated cancer. Cancer Res 2010;70:792801.

10. Werb Z. ECM and cell surface proteolysis: regulating cellular ecology. Cell 1997;91:439-442.

11. Miyazaki K, Hasegawa M, Funahashi K, Umeda M. A metalloproteinase inhibitor domain in Alzheimer amyloid protein precursor. Nature 1993;362:839-841.

12. Yu Q, Stamenkovic I. Cell surface-localized matrix metalloproteinase9 proteolytically activates TGF-beta and promotes tumor invasion and angiogenesis. Genes Dev 2000;14:163-176.

13. Li Q, Park PW, Wilson CL, Parks WC. Matrilysin shedding of syndecan-1 regulates chemokine mobilization and transepithelial efflux of neutrophils in acute lung injury. Cell 2002;111:635-646.

14. Ii M, Yamamoto H, Adachi Y, Maruyama Y, Shinomura Y. Role of matrix metalloproteinase-7 (matrilysin) in human cancer invasion, apoptosis, growth, and angiogenesis. Exp Biol Med 2006;231:20-27.

15. Miyazaki K, Hattori Y, Umenishi F, Yasumitsu H, Umeda M. Purification and characterization of extracellular matrix-degrading metalloproteinase, matrin (pump-1), secreted from human rectal carcinoma cell line. Cancer Res 1990;50:7758-7764.

16. Hasegawa S, Koshikawa N, Momiyama N, Moriyama K, Ichikawa Y, Ishikawa T, Mitsuhashi M, Shimada H, Miyazaki K. Matrilysinspecific antisense oligonucleotide inhibits liver metastasis of human colon cancer cells in a nude mouse model. Int J Cancer 1998;76:812-816.

17. Liver Cancer Study Group of Japan. General Rules for the Clinical and Pathological Study of Primary Liver Cancer, 2nd English edition. Tokyo: Kanehara, 2003.

18. Murakami Y, Uemura K, Sudo T, Hashimoto Y, Nakashima A, Kondo N, Sakabe R, Ohge H, Sueda T. Prognostic factors after surgical resection for intrahepatic, hilar, and distal cholangiocarcinoma. Ann Surg Oncol 2011;18:651-658.

19. Li YY, Li H, Lv P, Liu G, Li XR, Tian BN, Chen DJ. Prognostic value of cirrhosis for intrahepatic cholangiocarcinoma after surgical treatment. J Gastrointest Surg 2011;15:608-613.

20. DeOliveira ML, Cunningham SC, Cameron JL, Kamangar F, Winter JM, Lillemoe KD, Choti MA, Yeo CJ, Schulick RD. Cholangiocarcinoma: thirty-one-year experience with 564 patients at a single institution. Ann Surg 2007;245:755-762.

21. Saxena A, Chua TC, Sarkar A, Chu F, Morris DL. Clinicopathologic and treatment-related factors influencing recurrence and survival after hepatic resection of intrahepatic cholangiocarcinoma: a 19-year experience from an established Australian hepatobiliary unit. J Gastrointest Surg 2010;14:1128-1138.

22. Gruenberger B, Schueller J, Heubrandtner U, Wrba F, Tamandl D, Kaczirek K, Roka R, Freimann-Pircher S, Gruenberger T. Cetuximab, gemcitabine, and oxaliplatin in patients with unresectable advanced or metastatic biliary tract cancer: a phase 2 study. Lancet Oncol. 2010;11:1142-1148.

23. Ramanathan RK, Belani CP, Singh DA, Tanaka M, Lenz HJ, Yen Y, Kindler HL, Iqbal S, Longmate J, Mack PC, Lurje G, GandourEdwards R, Dancey J, Gandara DR. A phase II study of lapatinib in patients with advanced biliary tree and hepatocellular cancer. Cancer Chemother Pharmacol 2009;64:777-783.

24. Philip PA, Mahoney MR, Allmer C, Thomas J, Pitot HC, Kim G, Donehower RC, Fitch T, Picus J, Erlichman C. Phase II study of erlotinib in patients with advanced biliary cancer. J Clin Oncol 2006;24:3069-3074.

25. Zhu AX, Meyerhardt JA, Blaszkowsky LS, Kambadakone AR, Muzikansky A, Zheng H, Clark JW, Abrams TA, Chan JA, 
Enzinger PC, Bhargava P, Kwak EL, Allen JN, Jain SR, Stuart K, Horgan K, Sheehan S, Fuchs CS, Ryan DP, Sahani DV. Efficacy and safety of gemcitabine, oxaliplatin, and bevacizumab in advanced biliary-tract cancers and correlation of changes in 18fluorodeoxyglucose PET with clinical outcome: a phase 2 study. Lancet Oncol 2010;11:48-54.

26. Zhu AX, Hezel AF. Development of molecularly targeted therapies in biliary tract cancers: reassessing the challenges and opportunities. Hepatology 2011;53:695-704.

27. Valle J, Wasan H, Palmer DH, Cunningham D, Anthoney A, Maraveyas A, Madhusudan S, Iveson T, Hughes S, Pereira SP, Roughton M, Bridgewater J. Cisplatin plus gemcitabine versus gemcitabine for biliary tract cancer. N Engl J Med 2010;362:12731281.

28. Nelson AR, Fingleton B, Rothenberg ML, Matrisian LM. Matrix metalloproteinases: biologic activity and clinical implications. J. Clin. Oncol 2000;18:1135-1149.

29. Stetler-Stevenson WG, Hewitt R, Corcoran M. Matrix metalloproteinases and tumor invasion: from correlation and causality to the clinic. Semin Cancer Biol 1996;7:147-154.

30. Hanahan D, Weinberg RA. The hallmarks of cancer. Cell 2000;100:57-70.

31. Sawey ET, Johnson JA, Crawford HC. Matrix metalloproteinase 7 controls pancreatic acinar cell transdifferentiation by activating the Notch signaling pathway. Proc Natl Acad Sci U S A 2007;104:1932719332.

32. Wang WS, Chen PM, Wang HS, Liang WY, Su Y. Matrix metalloproteinase-7 increases resistance to Fas-mediated apoptosis and is a poor prognostic factor of patients with colorectal carcinoma. Carcinogenesis 2006;27:1113-20.

33. Mylona E, Kapranou A, Mavrommatis J, Markaki S, Keramopoulos A, Nakopoulou L. The multifunctional role of the immunohistochemical expression of MMP-7 in invasive breast cancer. APMIS 2005;113:246-255.

34. Mimori K, Yamashita K, Ohta M, Yoshinaga K, Ishikawa K, Ishii $\mathrm{H}$, Utsunomiya T, Barnard GF, Inoue H, Mori M. Coexpression of matrix metalloproteinase-7 (MMP-7) and epidermal growth factor (EGF) receptor in colorectal cancer: an EGF receptor tyrosine kinase inhibitor is effective against MMP-7-expressing cancer cells. Clin. Cancer Res 2004;10:8243-8249.

35. Kioi M, Yamamoto K, Higashi S, Koshikawa N, Fujita K, Miyazaki K. Matrilysin (MMP-7) induces homotypic adhesion of human colon cancer cells and enhances their metastatic potential in nude mouse model. Oncogene. 2003;22:8662-8670.

36. Tan $X$, Egami H, Ishikawa S, Sugita H, Kamohara H, Nakagawa $M$, Nozawa $F$, Abe $M$, Ogawa $M$. Involvement of matrix metalloproteinase-7 in invasion-metastasis through induction of cell dissociation in pancreatic cancer. Int J Oncol 2005;26;1283-1289.

37. Leelawat K, Narong S, Wannaprasert J, Ratanashu-ek T. Prospective study of MMP7 serum levels in the diagnosis of cholangiocarcinoma. World J Gastroenterol 2010;16:4697-4703.

38. Leelawat K, Sakchinabut S, Narong S, Wannaprasert J. Detection of serum MMP-7 and MMP-9 in cholangiocarcinoma patients: evaluation of diagnostic accuracy. BMC Gastroenterol 2009;9:30.

39. Itatsu K, Zen Y, Yamaguchi J, Ohira S, Ishikawa A, Ikeda H, Sato Y, Harada K, Sasaki M, Sasaki M, Sakamoto H, Nagino M, Nimura Y, Ohta T, Nakanuma Y. Expression of matrix metalloproteinase 7 is an unfavorable postoperative prognostic factor in cholangiocarcinoma of the perihilar, hilar, and extrahepatic bile ducts. Hum Pathol. 2008;39:710-719.

40. Miwa S, Miyagawa S, Soeda J, Kawasaki S. Matrix metalloproteinase7 expression and biologic aggressiveness of cholangiocellular carcinoma. Cancer 2002;94:428-434.

41. Jo Chae K, Rha SY, Oh BK, Koo JS, Kim YJ, Choi J, Park C, Park YN. Expression of matrix metalloproteinase-2 and -9 and tissue inhibitor of metalloproteinase- 1 and -2 in intraductal and nonintraductal growth type of cholangiocarcinoma. Am J Gastroenterol (2004) 99: 68-75.

42. Zhang C, Tu Z, Du S, Wang Y, Wang Q. Expression of matrix metalloproteinase 2 and extracellular matrix metalloproteinase inducer are unfavorable postoperative prognostic factors in intrahepatic cholangiocarcinoma. Pathol Oncol Res 2010;16:47-53.

43. Hung CF, Lu KC, Cheng TL, Wu RH, Huang LY, Teng CF, Chang WT. A novel siRNA validation system for functional screening and identification of effective RNAi probes in mammalian cells. Biochem Biophys Res Commun. 2006;346:707-20. 